\title{
Misexpression of Pou3f1 Results in Peripheral Nerve Hypomyelination and Axonal Loss
}

\author{
Elizabeth J. Ryu, ${ }^{1}$ James Y. T. Wang, ${ }^{1}$ Nam Le, ${ }^{1}$ Robert H. Baloh, ${ }^{2,3}$ Jason A. Gustin, ${ }^{1}$ Robert E. Schmidt, ${ }^{1,2}$ and \\ Jeffrey Milbrandt ${ }^{1,2,3}$ \\ ${ }^{1}$ Department of Pathology and Immunology, ${ }^{2} \mathrm{HOPE}$ Center for Neurological Disorders, and ${ }^{3}$ Department of Neurology, Washington University School of \\ Medicine, St. Louis, Missouri 63110
}

\begin{abstract}
Pou3f1/SCIP/Oct-6 is a POU-domain transcription factor that is an important regulator of peripheral nerve myelination by Schwann cells. Pou3f1-deficient mice experience a developmental delay in myelination indicating that transient induction of Pou3f1 is required for normal development of peripheral myelin. The mechanism by which Pou3f1 regulates myelination is unclear, because it can both increase expression of Egr2, a transcription factor that promotes the myelination program, and also repress the promoters of specific myelin genes such as myelin protein zero (MPZ) and myelin basic protein (MBP). Therefore, to investigate the effects of persistent Pou3f1 expression on peripheral nerve myelination, we created a conditional transgenic mouse [condPou3f1:MPZ(Cre)] that constitutively expresses Pou3f1 specifically in peripheral glia. Examination of sciatic nerves from condPou3f1:MPZ(Cre) mice revealed persistent hypomyelination and eventual axonal loss but no evidence of demyelination/remyelination processes or impaired Schwann cell proliferation. Nerves from these mice had normal levels of Egr2 mRNA but decreased levels of MPZ, MBP, and Pmp22 mRNA. Thus, unlike the Pou3f1 null mice, the condPou3f1:MPZ(Cre) mice exhibit persistent hypomyelination, indicating that strict control of Pou3f1 expression is critical to proper myelination. Our findings establish the importance of identifying factor(s) responsible for Pou3f1 downregulation during myelination, because they may play important roles in the development of peripheral neuropathies.
\end{abstract}

Key words: Pou3f1; Oct-6; SCIP; Egr2; myelination; neuropathy

\section{Introduction}

Myelin is essential for saltatory conduction of nerve signals in the peripheral nervous system. Persistent dysfunction of peripheral myelin eventually results in axonal degeneration, which manifests as limb weakness and sensory loss in diseases such as hereditary demyelinating neuropathies, also known as Charcot-Marie-Tooth (CMT) disease (Warner et al., 1999a; Houlden and Reilly, 2006). Currently, there are no effective medical therapies to treat or prevent these dysmyelinating diseases; therefore, a better understanding of the mechanisms controlling myelination are crucial. Several transcription factors, such as Egr2 (Krox-20), Sox10, and Pou3f1 (SCIP/Oct-6/Tst-1) have been implicated as important regulators of the myelination program (Jessen and Mirsky, 2002). Egr2, in complex with the Nab proteins, has been shown to regulate transcription of a number of essential myelin genes, and a mutation in the Egr2/Nab interaction domain is responsible for a severe form of hereditary neuropathy in humans

Received Dec. 19, 2006; revised July 27, 2007; accepted July 30, 2007.

This work was supported by National Institutes of Health (NIH) Neuroscience Blueprint Core Grant NS057105 to Washington University and the HOPE Center for Neurological Disorders, by NIH Grants NS040745 (J.M.), DK19645 (R.E.S.), AG10299 (R.E.S.), and 5-T32-DA07261 (E.J.R.), and by a grant by from the Juvenile Diabetes Research Foundation (R.E.S.). We thank Lawrence Wrabetz and Albee Messing for the MPZ(Cre) mice; Tatiana Gorodinsky, Nina Panchenko, Karen Green, Amy Strickland, and Amber Neilson for technical assistance; and members of the Milbrandt laboratory for helpful discussions and feedback on this manuscript.

Correspondence should be addressed to Jeffrey Milbrandt, Department of Neurology, Washington University School of Medicine, 660 South Euclid Avenue, Box 8118, St. Louis, M0 63110. E-mail: jmilbrandt@wustl.edu.

DOl:10.1523/JNEUROSCI.5497-06.2007

Copyright $\odot 2007$ Society for Neuroscience $\quad$ 0270-6474/07/2711552-08\$15.00/0
(Warner et al., 1998, 1999b; Le et al., 2005b). Mice deficient in Egr2, or those with null mutations in both Nab1 and Nab2, develop severe hypomyelination and die prematurely (Topilko et al., 1994; Le et al., 2005a,b). Sox10 also regulates transcription of genes critical for myelination, and Sox10 mutations are linked to hypomyelination in Waardenburg-Hirschsprung disease (Inoue et al., 1999; Peirano et al., 2000; Touraine et al., 2000; Ghislain and Charnay, 2006). However, Pou3f1 in particular has been an enigma, because it acts as both an activator and repressor of myelin gene transcription.

Pou3f1 is expressed in the developing nervous system, but in adulthood, expression is limited to the CNS, glial cells, and testis (He et al., 1989; Meijer et al., 1990; Blanchard et al., 1996). Pou3f1 expression in the murine peripheral nervous system is transient; it first appears in Schwann cell precursors at approximately embryonic day 14 (E14), peaks at approximately postnatal day 0 (P0), and is virtually undetectable in myelinating Schwann cells during adulthood (Blanchard et al., 1996; Zorick et al., 1996). However, low levels of Pou3f1 can be detected in nonmyelinating Schwann cells throughout life (Blanchard et al., 1996).

The initial functional characterization of Pou $3 \mathrm{f} 1$ revealed that it could bind and repress the myelin protein zero (MPZ) promoter, thus acting as a negative regulator of myelination, but subsequent studies found that Pou3f1 can also act as a positive regulator of myelination (Monuki et al., 1990; He et al., 1991). For instance, in Pou3f1 null mice, myelination was delayed at the promyelinating stage for $\sim 10 \mathrm{~d}$ before proceeding to form nor- 
mal myelin (Jaegle et al., 1996). Pou3f1 is also needed to fully induce Egr2 expression from the myelin Schwann cell enhancer (MSE), a cis-acting element in the Egr2 promoter responsible for the endogenous pattern of Egr2 expression (Ghislain et al., 2002). Recently, Pou3f1 has been shown to synergize with Sox10 to activate the MSE (Ghislain and Charnay, 2006).

Although numerous studies have examined the effects of Pou3f1 deficiency on myelination both in vitro and in vivo, the converse experiment in which Pou3f1 expression is aberrantly maintained during myelination has yet to be tested functionally. The transience of Pou3f1 expression during myelination suggests that it may also possess some inhibitory actions; therefore, proper myelination would depend on strict temporal control of Pou3f1. To address this hypothesis, we created a conditional Pou3f1 mouse that constitutively overexpresses Pou $3 \mathrm{f} 1$ specifically in peripheral glia. Characterization of this mouse mutant supports the idea that the downregulation of Pou3f1 may be essential for normal myelin development. In contrast to the Pou3f1 null mouse, continuous Pou3f1 overexpression in Schwann cells results in a permanent state of hypomyelination and significant secondary axonal loss, a point relevant to CMT1 neuropathies where hypomyelination leads to axonal loss and the subsequent limb weakness characteristic of these diseases. These results also stress the importance of understanding the mechanism of Pou3f1 regulation, because its temporal expression is critical for proper myelination of peripheral nerves.

\section{Materials and Methods}

Generation of condPou3f1 transgenic mice. The conditional activatable Pou3f1 construct was prepared using the DccRV vector, which contains the following essential elements. The chicken $\beta$-actin promoter is used to drive a floxed enhanced cyan fluorescent protein (ECFP) cassette. Downstream from this floxed cassette, a hemagglutinin (HA)-tagged mouse Pou3f1 cDNA is present followed by an internal ribosome binding site (IRES) and the fluorescent Venus reporter. The construct was electroporated into R1 ES cells, and ECFP-positive cells were selected and expanded. ES cells that expressed Pou3f1 after Cre-mediated excision of the floxed ECFP cassette were injected into BL6 blastocysts and implanted into pseudopregnant BL6 female mice. Southern blot analysis of tail DNA from founder animals identified by blue fluorescence in the skin taken by ear punch confirmed the presence of the conditional Pou3f1 allele. Routine genotyping was performed by screening ear punches for ECFP fluorescence and using primers that recognize Venus (5'ACCTGAGCTACCAGTCCGCCCTGAGCAA- $3^{\prime}$ ) and the polyadenylation sequence (5'-TCCTCATTAAAGGCATTCCACCA-3').

Primary rat Schwann cell culture, adenoviral infection, and transfection. Sciatic nerves from P1 or P2 rats were isolated using Brock's method and grown on collagen-coated plates in DMEM (Sigma, St. Louis, MO) containing 10\% FBS (Gemini Bio-Products, West Sacramento, CA), 2 mM glutamine, $2 \mu \mathrm{m}$ forskolin (Sigma), and $20 \mu \mathrm{g} / \mathrm{ml}$ bovine pituitary extract (Sigma) and penicillin/streptomycin as described previously (Nagarajan et al., 2001). Pou3f1-HA and green fluorescent protein (GFP) adenovirus were generated according to the AdEasy Vector System Application Manual (version 1.2; Qbiogene, Irvine, CA) and used to infect Schwann cells as described previously (Le et al., 2005a). In brief, adenovirus was added to Schwann cells in serum-free DMEM containing N2 supplement (Sigma) and $6 \mu \mathrm{m}$ forskolin for $24 \mathrm{~h}$, the cells were lysed, and RNA was purified for use in quantitative reverse transcription-PCR (qRT-PCR) assays. To establish the veracity of the conditional Pou3f1 construct, a six-well plate of human embryonic kidney 293T (HEK293T) cells was transfected with $1.25 \mu \mathrm{g}$ of Cre recombinase expression plasmid and 1.25 $\mu \mathrm{g}$ of DccRV or $1.25 \mu \mathrm{g}$ of Pou3f1-DccRV using calcium phosphate. Lysates were prepared after $48 \mathrm{~h}$, and Western blot analysis for HAPou3f1 was performed.

Quantitative RT-PCR. Trizol (Invitrogen, Carlsbad, CA) was used to isolate total RNA from Schwann cells and sciatic nerves. cDNA was tran- scribed from total RNA using Moloney murine leukemia virus reverse transcriptase (Invitrogen). Gene expression was quantified using qRTPCR performed using a SYBR green-based detection system on a 7700 Sequence Detector instrument (Applied Biosystems, Foster City, CA) as described previously (Nagarajan et al., 2001). Glyceraldehyde-3phosphate dehydrogenase expression was used to normalize samples and obtain relative expression values that were used to calculate fold changes. RNA was isolated from individual sciatic nerves of different mice, and at least two mice of each genotype were used to calculate an average relative expression level for each genotype.

Immunohistochemistry and Western blotting. For immunohistochemical analysis of sciatic nerve, the nerves were dissected and fixed in $4 \%$ paraformaldehyde overnight at $4^{\circ} \mathrm{C}$. For dorsal root ganglia (DRGs) analysis, P14 mice were transcardially perfused with PBS followed by $4 \%$ paraformaldehyde. DRGs were dissected, cleaned of extraneous tissue, and postfixed in $4 \%$ paraformaldehyde for one additional hour. For sciatic nerves and DRGs, sucrose cryoprotection was performed overnight followed by embedding in Tissue-Tek OCT Compound (Sakura Finetek, Torrance, CA) and cutting $8 \mu \mathrm{m}$ cryostat sections. For analysis of expression in the brain, fresh frozen OCT embedded brain was sectioned, and $8 \mu \mathrm{m}$ sections were fixed in $4 \%$ paraformaldehyde for $5 \mathrm{~min}$. For fluorescence immunohistochemistry, the sections were fixed for 2 min in $4 \%$ paraformaldehyde and then blocked in $10 \%$ horse serum for $1 \mathrm{~h}$ at room temperature. Incubation with primary antibody was performed overnight at $4^{\circ} \mathrm{C}$, and secondary antibody incubation was performed at room temperature for $1 \mathrm{~h}$. For HA immunohistochemistry, the sections were fixed for $2 \mathrm{~min}$ in $4 \%$ paraformaldehyde, blocked in 3\% milk/TBS for $1 \mathrm{~h}$, and incubated with anti-HA antibody (1:5000) (see below) diluted in blocking buffer overnight at $4^{\circ} \mathrm{C}$. Subsequent steps were performed using the Vectastain Elite ABC Kit from Vector Laboratories (Burlingame, CA). Slides were developed using a DAB peroxidase substrate (Sigma). For Western immunoblotting, transfected HEK293T cells were lysed in $2 \times$ Laemmli buffer, and sciatic nerves were homogenized in lysis buffer ( $0.25 \mathrm{~m}$ Tris- $\mathrm{HCl}$, pH 6.8, 20\% glycerol, $4 \%$ SDS, $10 \%$ $\beta$-mercaptoethanol). The protein lysates were fractionated on $10 \%$ SDSPAGE and transferred to nitrocellulose. The primary antibodies used were rabbit anti-Pou3f1 (1:1000; kind gift from J. Bermingham, McLaughlin Research Institute, Great Falls, MT), rabbit anti-HA (1:5000) (see below), and rabbit anti- $\beta$-actin (1:1000; Cell Signaling Technology, Danvers, MA). Secondary antibodies used were anti-rabbit Cy3 (1:1500), anti-rabbit Alexa 488 (1:1500), anti-rabbit HRP (1:5000; all from Jackson ImmunoResearch, West Grove, PA). The affinitypurified polyclonal HA antibody was generated by immunizing a rabbit with the synthetic peptide CYPYDVPDYASL (Pacific Immunology, Ramona, CA). When necessary, immunoblots were stripped in a stripping buffer (62.5 mM Tris- $\mathrm{HCl}, \mathrm{pH} \quad 6.7)$ containing $2 \%$ $\beta$-mercaptoethanol for $30 \mathrm{~min}$ at $50^{\circ} \mathrm{C}$ before reanalysis.

Light and electron microscopy. Mice were transcardially perfused with Karnovsky's fixative, and sciatic nerves were postfixed in Karnovsky's fixative for an additional $16 \mathrm{~h}$. Nerve segments were dehydrated in graded alcohols and embedded in Epon-Araldite. One-micrometer-thick plastic embedded sections were prepared and toluidine blue stained for light microscopy. For electron microscopy, thin sections were prepared, stained with uranyl acetate and lead citrate, and photographed with a JEOL (Akishima, Japan) 1200 electron microscope. For spinal cord and femoral motor and sensory nerves, mice were fixed with $4 \%$ paraformaldehyde and then spinal cord and nerves were removed and washed in PBS. Spinal cord and nerves were incubated in $1 \%$ osmium tetraoxide containing $1.5 \%$ potassium ferricyanide in $0.5 \mathrm{~m}$ phosphate buffer for $\sim 1$ $\mathrm{h}$. Tissues were washed in PBS and embedded in paraffin, and 1- $\mu \mathrm{m}-$ thick sections were cut and stained with toluidine blue.

Osmicated teased sciatic nerve fibers. Mice were transcardially perfused with Karnovsky's fixative and sciatic nerves postfixed in Karnovsky's fixative for an additional $16 \mathrm{~h}$. Nerves were washed first with deionized water, followed by PBS, and then incubated in $1 \%$ osmium tetraoxide plus $1.5 \%$ potassium ferricyanide in $0.5 \mathrm{M}$ phosphate buffer for $\sim 1 \mathrm{~h}$. Nerves were washed in PBS followed by incubation in 33, 66, and $100 \%$ glycerol/PBS for $\sim 6 \mathrm{~h}$ each. Nerves were teased in $100 \%$ glycerol and coverslipped, and photomicrographs were taken. 
g-ratio, axon counts, and axon diameter measurements. g-ratios were calculated by dividing the axon diameter by the total diameter of the myelinated axon as measured from digital electron micrographs. One hundred g-ratios from two animals from each genotype at each age were used to calculate an average $g$-ratio. For axon counts, axons $>1 \mu \mathrm{m}$ in diameter were counted from two digital electron micrographs from two animals of each genotype at each age to achieve axons $/ \mathrm{mm}^{2}$. The total area of the nerves was then calculated from toluidine blue plastic sections to calculate the average total number of axons per nerve. To calculate percentage of axons, the total number of axons per nerve in condPou $3 \mathrm{f} 1$ : $\mathrm{MPZ}$ (Cre) mice was divided by the average total number of axons per nerve in control mice. For axon diameter, 100 axon diameters were measured from two digital electron micrographs from two animals from each genotype, and the average axon diameter was calculated. NIH ImageJ software was used to aid in measurements and counting.

Statistics. Except for the Kolmogorov-Smirnov test, all $p$ values were calculated using a two-tailed Student's $t$ test.

\section{Results}

\section{Generation of Pou3f1 transgenic mice}

To study the effects of aberrant Pou3f1 expression during myelination, conditional transgenic mice were produced that constitutively drive expression of Pou3f1 in peripheral glia. This was achieved using a binary system with a conditional floxed Pou3f1 allele that could be activated by Cre recombinase. The conditional Pou3f1 (condPou3f1) construct was designed to express the fluorescent ECFP reporter via the chicken $\beta$-actin promoter in the absence of Cre recombinase. In the presence of Cre, the floxed ECFP cassette is excised allowing expression of the HAtagged Pou3f1 cDNA and the fluorescent Venus reporter that was placed downstream of the IRES (Fig. $1 A$ ). When this conditional construct was cotransfected into HEK293T cells along with a vector expressing Cre recombinase, Pou3fl expression was detected by immunoblotting using both Pou3f1 and HA antibodies (Fig. $1 B$ ). In the absence of Cre recombinase, Pou3f1 expression is undetectable, thus demonstrating the tight control of Pou3f1 expression achieved with the use of this conditional system.

Embryonic stem cells were electroporated with this conditional Pou3f1 vector, and colonies expressing the ECFP reporter were picked for additional analysis. Cells in which Pou3f1 expression could be activated by Cre recombinase were injected into blastocysts, and transgenic mice (named condPou3f1) containing the conditional allele were obtained (see Materials and Methods). To constitutively express Pou3f1 in Schwann cells, the condPou3f1 mice were mated to MPZ(Cre) mice, which express Cre recombinase in peripheral glia (Feltri et al., 1999, 2002), to produce condPou3f1:MPZ(Cre) mice. In these mice, the ECFP cassette is excised and Pou3f1-HA expression is activated specifically in sciatic nerve as detected by Western immunoblotting using Pou3f1 and HA antibodies (Fig. 1C). Immunostaining for Pou3f1 in 4-month-old (supplemental Fig. $1 A$, available at www. jneurosci.org as supplemental material) and 6-month-old (Fig. $1 E)$ condPou3f1:MPZ(Cre) sciatic nerve demonstrated a large number of Schwann cells that expressed elevated levels compared with wild type, whereas there was no difference in Pou3f1 staining between wild-type and condPou3f1:MPZ(Cre) cerebral cortex (Fig. $1 F$ ), dorsal root ganglia neurons (supplemental Fig. $1 B$, available at www.jneurosci.org as supplemental material), or other tissues (data not shown). The presence of exogenously expressed HA-tagged Pou3f1 in condPou3f1:MPZ(Cre) sciatic nerve at P14 was confirmed by immunohistochemistry using an anti-HA antibody (supplemental data Fig. $1 C$, available at www. jneurosci.org as supplemental material). These results indicate that the MPZ-driven Cre expression of Pou3f1 was indeed tar- geted to peripheral glia. We used qRT-PCR to further characterize Pou3fl overexpression in the sciatic nerve and found that Pou3f1 mRNA levels were sixfold higher in P14 condPou3f1: MPZ(Cre) sciatic nerve compared with wild-type or condPou3f1 transgenic sciatic nerve (Fig. $1 D$ ) and 15-fold higher in P28 condPou3f1:MPZ(Cre) sciatic nerve compared with control (supplemental Fig. $1 D$, available at www.jneurosci.org as supplemental material). These data demonstrate that expression from the conditional Pou3f1 allele is not leaky and that Pou3f1 is overexpressed only in Schwann cells of the condPou3f1:MPZ(Cre) mice.

\section{Constitutive Pou3f1 overexpression leads to persistent hypomyelination and axonal loss in the peripheral nervous system}

The condPou3f1:MPZ(Cre) mice are indistinguishable from their control littermates at birth, and most of these animals survive until at least 10 months of age [the longest a condPou3f1: $\mathrm{MPZ}(\mathrm{Cre})$ mouse was allowed to survive before it was killed]. Although the condPou3f1:MPZ(Cre) mice are of similar weight at birth as control littermates, they do not grow normally, and by 6 months of age, they weigh $40 \%$ less than their wild-type counterparts (Fig. $2 A, B$ ). By 3 weeks of age, the condPou3f1: MPZ(Cre) mice appear weak and begin to drag their hindlegs when they move, a posture that is characteristic of mice with muscle weakness caused by peripheral neuropathy (supplemental movie 1, available at www.jneurosci.org as supplemental material). The condPou3f1:MPZ(Cre) mice also exhibit abnormal limb clasping, mild tremors, and impaired coordination (supplemental movie 2, available at www.jneurosci.org as supplemental material), similar to that seen in other mice with deficits in peripheral myelination (Suter et al., 1992; Le et al., 2005a,b). Because myelopathy in the spinal cord could also result in the motor disturbances we observed, we examined 1- $\mu \mathrm{m}$-thick paraffin sections of the spinal cord of the condPou3fi:MPZ(Cre) mice. We found that the spinal cord was indistinguishable from control sections indicating that the motor deficits of the condPou3f1: MPZ(Cre) mice are caused by peripheral myelination deficiencies (Fig. 2C).

During late embryogenesis, myelination begins as Schwann cell precursors that have migrated along axons and extended processes to engulf bundles of axons begin to select single axons for myelination. At birth, these promyelinating Schwann cells establish a 1:1 relationship with axons and begin to myelinate them. These early myelinating Schwann cells express relatively high levels of Pou3f1 and begin to express Egr2. To examine the effect of Pou3f1 overexpression in Schwann cells on peripheral nerve myelination, we examined sciatic nerves from wild-type and condPou3f1:MPZ(Cre) mice. After gross examination, the sciatic nerve of 6-month-old condPou3f1:MPZ(Cre) mice revealed that the nerve was thinner and less opaque than nerves taken from wild-type mice, suggesting that overexpression of Pou3f1 in Schwann cells leads to myelin abnormalities (supplemental Fig. $2 A, B$, available at www.jneurosci.org as supplemental material). Microscopic examination of plastic-embedded $1-\mu \mathrm{m}$-thick sciatic nerve sections from condPou3f1:MPZ(Cre) mice at P14 and 6 months of age confirmed abnormal myelination with many axons containing no myelin or very thin myelin sheaths (Fig. 2D).

Electron microscopic examination of condPou3f1:MPZ(Cre) sciatic nerve already showed evidence of hypomyelination even in the early stages of myelination at P4 (Fig. 3A-D). By P14, when normal myelination is primarily complete, several Schwann cells 
A.
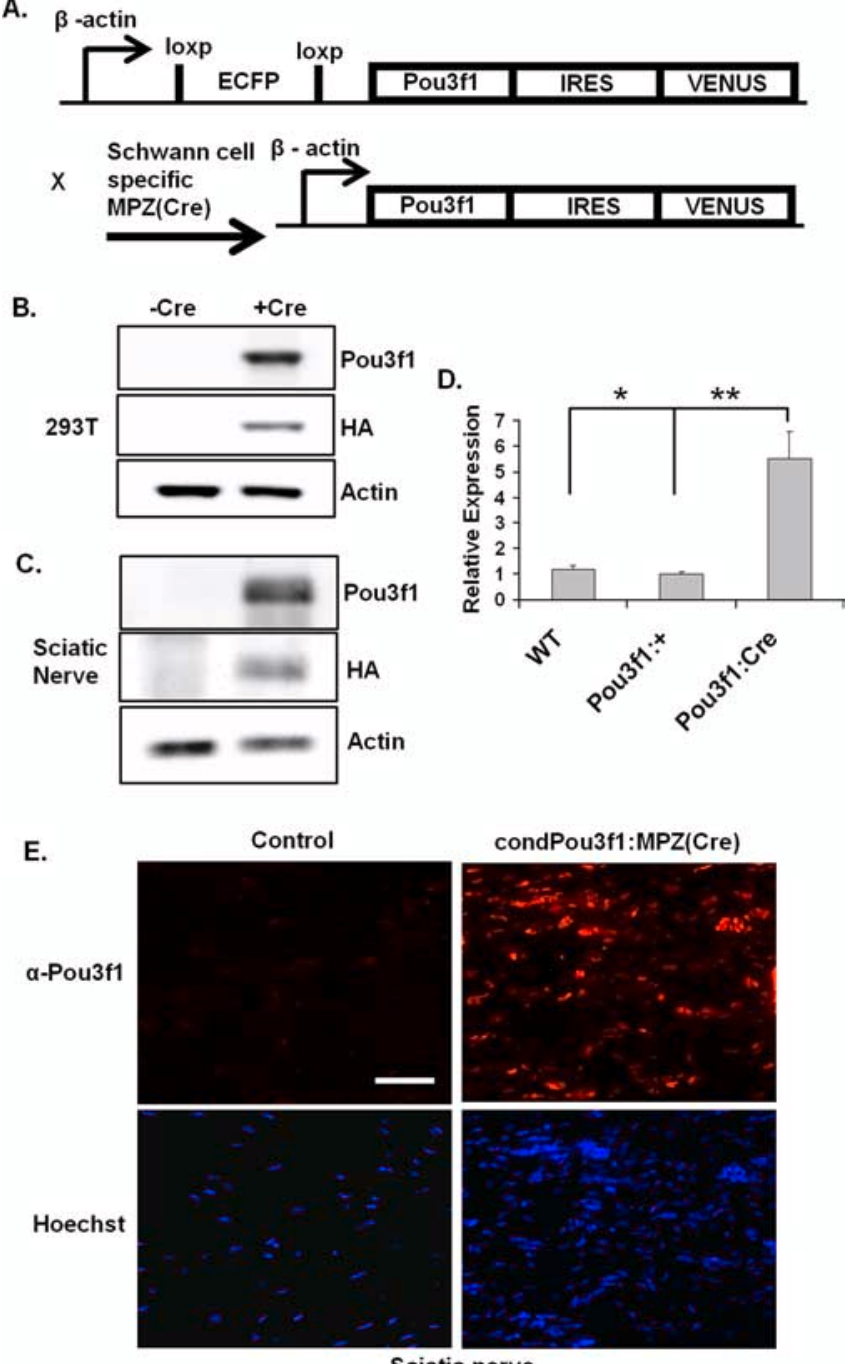

Sciatic nerve

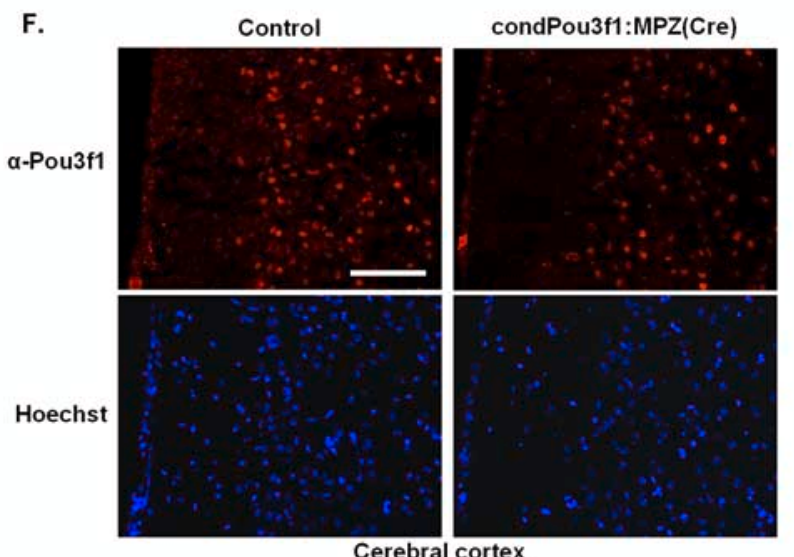

Cerebral cortex

Figure 1. Generation of condPou3f1:MPZ(Cre) mice. A, Diagram of condPou3f1 construct (see Materials and Methods for details). When Pou3f1 transgenic mice are crossed with mice that express Cre recombinase under control of the MPZ promoter, the ECFP cassette is excised, allowing for Pou3f1 and Venus expression specifically in Schwann cells. B, In HEK293T cells, cotransfection of the condPou3f1 construct and Cre recombinase results in Pou3f1 expression as detected by immunoblotting using Pou3f1 and $\mathrm{HA}$ antibodies. The conditional vector is not leaky as in the absence of Cre recombinase; no Pou3f1-HA expression is detected. The blot was stripped and reprobed with $\beta$-actin to control for protein loading. Comparable results were achieved in an independent experiment. C, Immunoblotting showed elevated Pou3f1-HA protein levels in sciatic nerve lysate from 6-month-old condPou3f1:MPZ(Cre) ( + Cre) versus control (-Cre) mice. The blot was stripped and reprobed with $\mathrm{HA}$ and $\beta$-actin to control for protein loading. Similar results were achieved in an independent experiment. $\boldsymbol{D}$, qRT-PCR showed in the condPou3f1:MPZ(Cre) nerves were stalled at only a single wrap of the axon, whereas in wild-type nerves, there were many more abundantly myelinated axons (Fig. $3 E-H$ ). Of the axons that were myelinated, many possessed a myelin sheath that was significantly thinner than normal (Fig. $3 E-H$; Table 1). A similar nerve phenotype is apparent at all other ages examined, including P28 and 4, 6, and 10 months (Fig. 3I-L; Table 1) (supplemental Fig. 2C, available at www.jneurosci.org as supplemental material). Because differences in myelination between motor and sensory nerves have been observed in other models of hereditary neuropathy (Scherer et al., 1998), we examined the motor and sensory branches of the femoral nerve. $1-\mu \mathrm{m}$-thick paraffin sections of the femoral motor and sensory nerves of condPou3f 1 : $\mathrm{MPZ}(\mathrm{Cre})$ mice also showed hypomyelination similar to the sciatic nerves from these mice (supplemental Fig. $2 D$, available at www.jneurosci.org as supplemental material). Additionally, analysis revealed that the extent of hypomyelination appeared to be equivalent in the motor and sensory nerves in the condPOU3f1:MPZ(Cre) mice, indicating there is no motor versus sensory nerve bias in this hypomyelinating mouse model. Although the vast majority of axons in the condPou3fl: $\mathrm{MPZ}(\mathrm{Cre})$ nerves were hypomyelinated, there were occasional normally myelinated axons present even in aged adult animals, which are probably the result of incomplete Cre activation of the conditional Pou3f1 transgene.

To further characterize the myelination defects caused by Pou3f1 overexpression, we calculated the g-ratio distribution (axon diameter divided by total myelinated axon diameter) at both P14 and 6 months of age in mutant and wild-type animals. This analysis indicated that many Schwann cells in the condPou3f1:MPZ(Cre) mice stall at one wrap, and that there is a much higher number of hypomyelinated axons ( $g$-ratio closer to $1.0)$ in the condPou3f1:MPZ(Cre) mice (Table 1). Many of the hypomyelinated axons are myelinated by Schwann cells that possess an enlarged nucleus, whereas in the control nerves, most Schwann cell nuclei are compacted and are not clearly visible (Fig. $3 I, J)$. Both large and small caliber axons are hypomyelinated in apparently equal numbers. We observed similar deficits and significant hypomyelination in condPou $3 \mathrm{f} 1: \mathrm{MPZ}(\mathrm{Cre})$ mice even at 6 and 10 months of age (Fig. 3I-L; Table 1) (supplemental Fig. 2C, available at www.jneurosci.org as supplemental material). Thus, in contrast to the Pou3f1 null animals, the hypomyelination caused by persistent Pou3f1 expression continues throughout the life of these animals. Constitutive Pou3f1 expression through adulthood did not result in irregular cell cycle progression, because there was no aberrant Ki67 expression (data not shown). In addition, there was no evidence of onion bulb remyelination (Fig. 3A-L) (supplemental Fig. 2C, available at www. jneurosci.org as supplemental material). Examination of osmicated teased fibers from 6-month-old condPou3f1:MPZ(Cre) sciatic nerves indicated the presence of Wallerian degeneration and small amounts of myelin debris (Fig. 3M), segmental demyelination, and multiple Schwann cell nuclei on unmyelinated segments (Fig. $3 N$ ).

\section{$\leftarrow$}

sixfold higher levels of Pou3f1 mRNA in sciatic nerve from P14 condPou3f1:MPZ(Cre) $(n=3$ mice) compared with wild-ype mice (WT) ( $n=3$ mice) or condPou3f1 transgenic mice ( $n=4$ mice). Values reported are the means \pm SEM ( $\left.{ }^{*} p=0.02 ;{ }^{* *} p=0.004\right) . E, F$, Immunostaining of sciatic nerve from 6-month-old condPou3f1:MPZ(Cre) mice revealed high Pou3f1 expression compared with control wild-type mice. № ectopic Pou3f1 expression was present in the cerebral cortex of condPou3f1:MPZ(Cre) mice compared with wild-type control, indicating the peripheral glia-specific expression of Pou3f1 in condPou3f1:MPZ(Cre) mice. Scale bar, $100 \mu \mathrm{m}$. 
A.

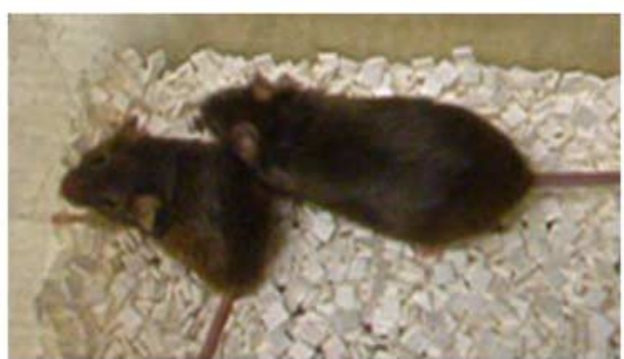

B.

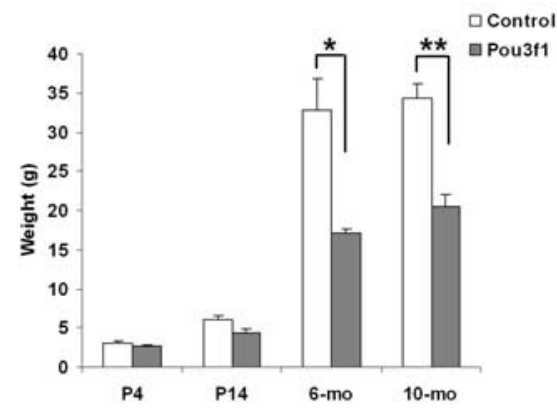

c.

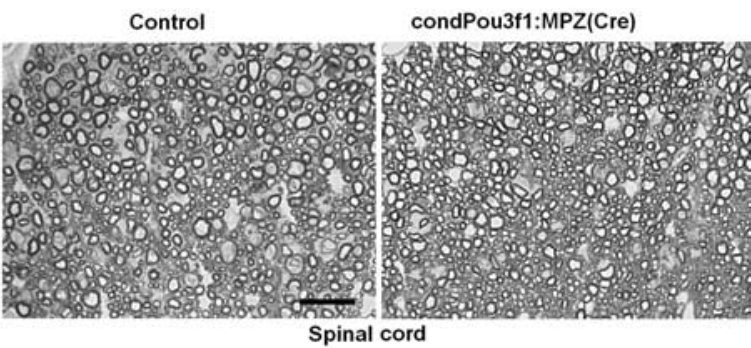

D.

Control

condPou3f1:MPZ(Cre)

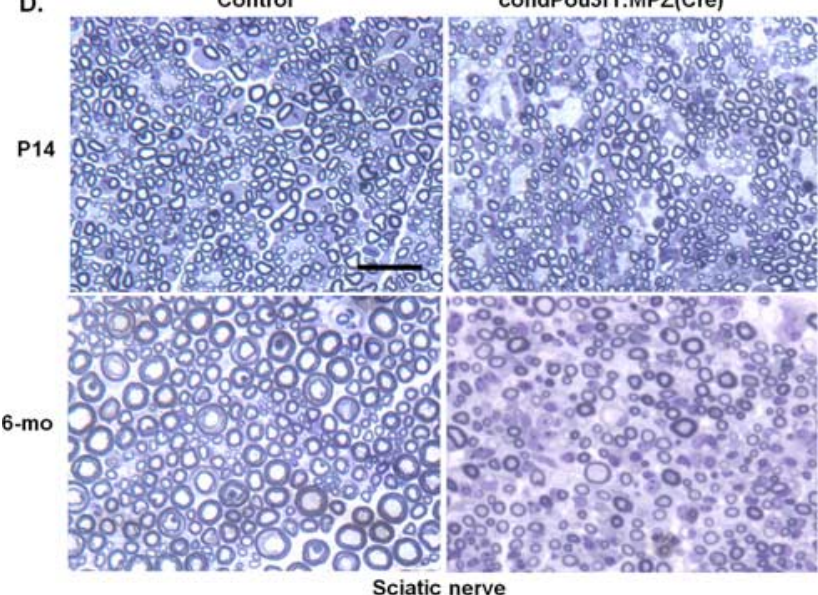

Sciatic nerve

Figure 2. Characterization of condPou3f1:MPZ(Cre) mice. $\boldsymbol{A}$, Photograph of 6-month-old wild-type and condPou3f1:MPZ(Cre) mice demonstrates the decreased size of the mutant mouse (left). $\boldsymbol{B}$, Weight comparison graph of control versus condPou3f1:MPZ(Cre) mice at indicated ages. At birth, condPou3f1:MPZ(Cre) mice are indistinguishable from control littermates, however, as the mice age, the body weight differences become profound. Values represent average $\pm \operatorname{SEM}\left(n=\right.$ at least 2 animals of each genotype at each time point; ${ }^{*} p=0.02 ;{ }^{* *} p=$ 0.0005). C, One-micrometer-thick paraffin sections of spinal cord from condPou3f1:MPZ(Cre) and control mice show that there is no difference in myelination of the CNS as evident from digital photographs of the ventral funiculi. Scale bar, $40 \mu \mathrm{m}$. D, Light microscopy of toluidine blue stained 1- $\mu \mathrm{m}$-thick plastic sections show decreased numbers of myelinated axons in condPou3f1:MPZ(Cre) versus wild-type nerves. Scale bar, $40 \mu \mathrm{m}$.

Evidence of Wallerian degeneration from osmicated teased fibers and examination of electron micrographs of sciatic nerves from 6- and 10-month-old condPou3f1:MPZ(Cre) mice suggested that there was secondary axonal degeneration occurring in these mice (Fig. 3I-J,M) (supplemental Fig. 2C, available at www. jneurosci.org as supplemental material). To explore this possibility, we counted the number of axons in the sciatic nerves of these animals. We found that there were significantly less axons in the condPou3f1:MPZ(Cre) sciatic nerves at both 6 months (52\% less) and 10 months ( $84 \%$ less) (Fig. 4A). The number of axons in P4 mutant animals was normal, indicating that the decreased axon number was a result of axonal loss and not a developmental defect reflected as a decrease in axon number (Fig. 4A). We also found that the diameter of the axons was similar in condPou3f1: $\mathrm{MPZ}(\mathrm{Cre})$ and wild-type sciatic nerves at P4, 6 months, and 10 months (Fig. $4 B$ ), indicating that condPou3f1:MPZ(Cre) mice undergo an age-dependent loss of both large and small caliber axons most likely a result of significant and prolonged hypomyelination.

Constitutive Pou3f1 expression leads to aberrant myelin gene expression despite normal Egr2 levels

The persistent hypomyelination of the condPou3f1:MPZ(Cre) sciatic nerve led us to examine whether constitutive expression of Pou3f1 leads to abnormal expression of key myelination genes that would in turn result in defective myelination. Pou3f1 is a member of a family of transcription factors that contain a POU domain. The POU domain is composed of two parts, the POUspecific domain and the POU-homeodomain, that function cooperatively to bind DNA (Herr and Cleary, 1995). Pou3f1 aids in the induction of Egr2, a transcription factor essential for regulating expression of several important myelin proteins (Ghislain et al., 2002). Pou3f1 null mice exhibit delayed activation of Egr2, and recent studies have reported that Pou3f1 can synergize with Sox10 to induce Egr2 (Ghazvini et al., 2002; Ghislain and Charnay, 2006). Thus, we analyzed the expression of Egr2 in P14 condPou3f1:MPZ(Cre) sciatic nerves compared with control nerves. qRT-PCR revealed that Egr2 $\mathrm{mRNA}$ levels were similar in the condPou3f1:MPZ(Cre) nerves compared with control (Fig. 5A). We next examined levels of the myelin structural proteins MPZ and MBP, because Pou3f1 acts as a transcriptional repressor of these promoters (Monuki et al., 1993). condPou3f1:MPZ(Cre) nerves contained lower mRNA levels of MPZ, MBP, and Pmp22 compared with control nerves, whereas levels of $\mathrm{Nab} 2$ and periaxin remained unchanged (Fig. 5A). To confirm these findings, we infected cultured rat Schwann cells with adenovirus expressing Pou3f1 and stimulated myelin gene expression by adding $6 \mu \mathrm{m}$ forskolin to the media and examined the levels of Egr2, MPZ, and Pmp22 mRNAs. Similar to our in vivo results, we found that Pou3f1 overexpression did not increase Egr $2 \mathrm{mRNA}$ levels. However, it did result in reduced MPZ and Pmp22 mRNA levels (Fig. 5B). Together, these results are consistent with previous reports of Pou3f1 acting as a transcriptional repressor of MPZ and MBP and indicate that the primary defect in the condPou3f1:MPZ(Cre) mice is not abnormal expression of Egr2/Nab2 complexes but rather that constitutive Pou3f1 expression inhibits expression of multiple myelin proteins that then leads to aberrant myelin formation.

\section{Discussion}

To investigate the role of the Pou3f1 homeodomain protein in peripheral nerve myelination, we generated and characterized mice that conditionally overexpress Pou3f1 in Schwann cells in response to Cre recombinase activation. Our results show that, unlike the transitory delay in myelination observed in Pou3f1 null mice, constitutive Pou3f1 expressing mice have persistent hypomyelination of both peripheral motor and sensory axons and eventual axonal loss. Interestingly, CNS myelin deficiencies develop 

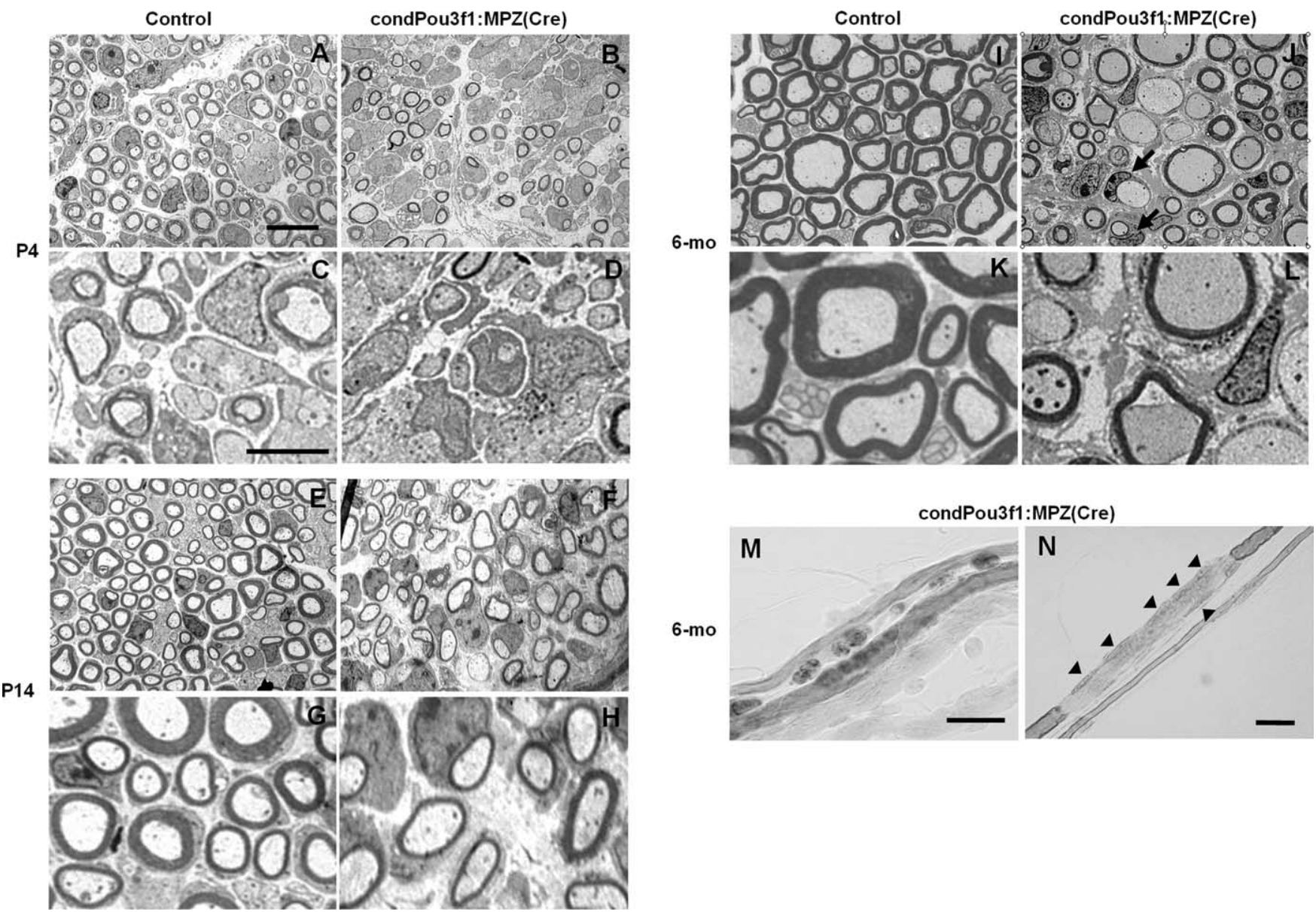

Figure 3. Constitutive Pou3f1 expression leads to persistent hypomyelination and axonal loss. $A, B, E, F, I, J$, Ultrastructural analysis of control and condPou3f1:MPZ(Cre) nerves show that condPou3f1:MPZ(Cre) nerves are hypomyelinated as early as $\mathrm{P} 4$ and remain so at 6 months of age. Scale bar, $10 \mu \mathrm{m}$. The arrows in $J$ indicate the clearly visible enlarged nuclei of the mutant $S c h w a n n$ cells compared with the undetectable compacted nuclei of the control Schwann cells. C, D, G, H, $\boldsymbol{K}, \boldsymbol{L}$, Higher-magnification electron microscopy images of control and condPouzf1:MPZ(Cre) sciatic nerves. Scale bar, $5 \mu \mathrm{m}$. Osmicated teased fibers from condPou3f1:MPZ(Cre) sciatic nerve shows axonal degeneration and myelin debris $(\boldsymbol{M})$ and segmental demyelination and multiple Schwann cell nuclei ( $\boldsymbol{N}$, arrowheads) on unmyelinated segments. Scale bar, $20 \mu \mathrm{m}$.

Table 1. g-ratios of control and condPou3f1:MPZ(Cre) sciatic nerves

\begin{tabular}{llll}
\hline & Control & condPou3f1: & \\
& MPZ(Cre) & Statistics \\
\hline P14 & $0.658 \pm 0.069$ & $0.775 \pm 0.127$ & $D=0.4898$ \\
& Range, $0.846-0.455$ & Range, $1.00-0.517$ & $p<0.001$ \\
6 Month & $0.648 \pm 0.077$ & $0.769 \pm 0.119$ & $D=0.4975$ \\
& Range, $0.856-0.427$ & Range, $1.00-0.483$ & $p<0.001$ \\
\hline
\end{tabular}

$g$-ratio values (diameter of axon divided by the total diameter of axon and myelin sheath) of P14 and 6-month-old condPou3f1:MPZ(Cre) mice are larger, indicating thinner myelin sheaths. Data were analyzed using the KolmogorovSmirnov test, and $g$-ratios shown are mean \pm SD.

in transgenic mice that overexpress Pou3f1 in oligodendrocytes under control of the MBP promoter (Jensen et al., 1998). However, unlike in our model, the authors observed hypermyelination and premature myelin formation in addition to hypomyelination and could not detect any differences in mRNA expression of MBP or PLP (proteolipid protein) in the brains of the transgenic mice, suggesting that the mechanism causing myelin disruption in that model may differ from that of the condPou3f1:MPZ(Cre) transgenic mice.

Examination of Egr2 mRNA levels in P14 condPou3f1: $\mathrm{MPZ}(\mathrm{Cre})$ nerves revealed that levels were normal compared with controls and implies that elevated or aberrant Pou3f1 expression does not induce higher levels of Egr2 mRNA. This is in contrast with previous reports in which Pou3f1 appears to activate Egr2 induction (Ghislain et al., 2002; Ghislain and Charnay,
2006). It now appears that Pou3f1 synergizes with Sox 10 to regulate Egr2 expression, thus one possible explanation for this discrepancy could be that Egr2 regulation requires a strict stoichiometric ratio of Pou3f1 to Sox10, or possibly that another coactivator is altered by the overexpression of Pou3f1.

Our finding that the mRNA levels of myelin proteins such as $\mathrm{MPZ}$ and MBP were decreased in vivo in nerves from condPou3f1:MPZ(Cre) mice supports previous in vitro data showing Pou3f1 inhibits transcription from the MPZ promoter (Monuki et al., 1993). Of note, levels of Pmp22 were also lower in condPou3f1:MPZ(Cre) sciatic nerve suggesting that Pou3f1 may have multiple targets of repression similar to the ability of Egr2 to initiate transcription of multiple target myelin genes. Interestingly, condPou3f1:MPZ(Cre) Schwann cells expressed lower levels of MPZ, MBP, and Pmp22 mRNAs even in the presence of normal Egr2 levels, suggesting that Pou3f1 activity predominates over Egr2 activity in regulating their expression. Together, it appears that the primary hypomyelination defect caused by constitutive Pou3fl expression in peripheral glia is not mediated through abnormal Egr2 regulation but is directly exerted at the level of myelin protein gene regulation.

Despite the involvement of Pou3f1 in regulating Egr2 expression, its function in this regard can be fulfilled by other POU domain transcription factors, such as Brn-1 and Brn-2. The abil- 
ity of Brn-1 and Brn-2 to compensate for the loss of Pou3f1 was demonstrated using mutant knock-in mice in which Brn-1 (or Brn-2) was inserted into the Pou3f1 locus effectively replacing Pou3f1 expression. The Brn-1 knock-in mice displayed normal Egr2 expression and onset of myelination, whereas Brn-2 knock-in mice manifested a partial rescue of the aberrant myelination phenotype present in Pou3f1-deficient mice (Jaegle et al., 2003; Friedrich et al., 2005). These findings indicate that Brn-1 and Brn-2 are functionally capable of rescuing the myelination delay of the Pou3f1 null mice and provide additional evidence that, as previously hypothesized, Pou3f1 is required for the initiation of myelination (Jaegle and Meijer, 1998; Zorick et al., 1999).

Although its been shown that Brn-1 and Brn-2 can compensate for Pou3f1 deficiency to prevent the transient defects in myelination observed in Pou3f1-deficient mice, there appears to be no similar compensatory strategy capable of rescuing the hypomyelination resulting from Pou3f1 overexpression as hypomyelination persists in the condPou3f1:MPZ(Cre) model. These results stress the importance of defining the mechanism and identifying the factor(s) responsible for the temporal expression of Pou3f1 in normal Schwann cell development, because persistent Pou3f1 expression leads to hypomyelination and severe axonal loss. One possible candidate for this role is Pou3f1 itself, because in mice harboring a Pou3f1 null allele containing a LacZ reporter, the LacZ expression from the Pou3f1 locus persisted even in adulthood (Jaegle and Meijer, 1998). In addition, Pou3f1 autorepression concurs with the hypothesis that Pou3f1 acts as a transcriptional repressor during myelination as evidenced here by the repression of myelin gene expression in condPou3 $\mathrm{fl}$ : $\mathrm{MPZ}(\mathrm{Cre})$ nerves. Another possible candidate for Pou3f1 downregulation is the Egr2/Nab protein complex, because Pou3f1 expression is aberrantly maintained in Schwann cells from Egr2 null, Egr ${ }^{\text {Lo/Lo }}$, and Nab double knock-out mice (Zorick et al., 1999; Le et al., 2005a,b).

The exact role of Pou3f1 during myelination has been unclear for some time because of the apparently contradictory phenotypes of the Pou3f1 null mouse (hypomyelination) compared with a dominant-negative Pou3f1 transgenic mouse (hypermyelination) and by its ability to activate Egr2 expression while repressing expression of Egr2 targets such as MPZ (Monuki et al., 1993; Weinstein et al., 1995; Bermingham et al., 1996; Jaegle et al., 1996; Ghazvini et al., 2002; Ghislain et al., 2002). Seemingly contradictory to this paradigm, evidence from two studies suggests a possible explanation for these contradictory phenotypes. Ghazvini et al. (2002) show that in the sciatic nerve of their Pou3f1deficient mice, there are lower Egr2 and MPZ protein levels [which does not reflect mRNA status, because the MPZ protein can be shuttled to the lysosome under nonfavorable myelination conditions resulting in low protein levels (Brunden and Poduslo, 1987)], whereas Bermingham et al. (1996) showed normal MPZ and MBP mRNA levels but did not measure Egr2 levels in their Pou3f1-deficient mice (Bermingham et al., 1996; Ghazvini et al., 2002). Together, these data suggest that Pou3f1-deficient mice could have low protein levels of Egr2, MPZ, and MBP in the midst of normal mRNA levels of MPZ and MBP. A possible rationale for this scenario is that the lower Egr2 levels are a direct result of Pou3f1
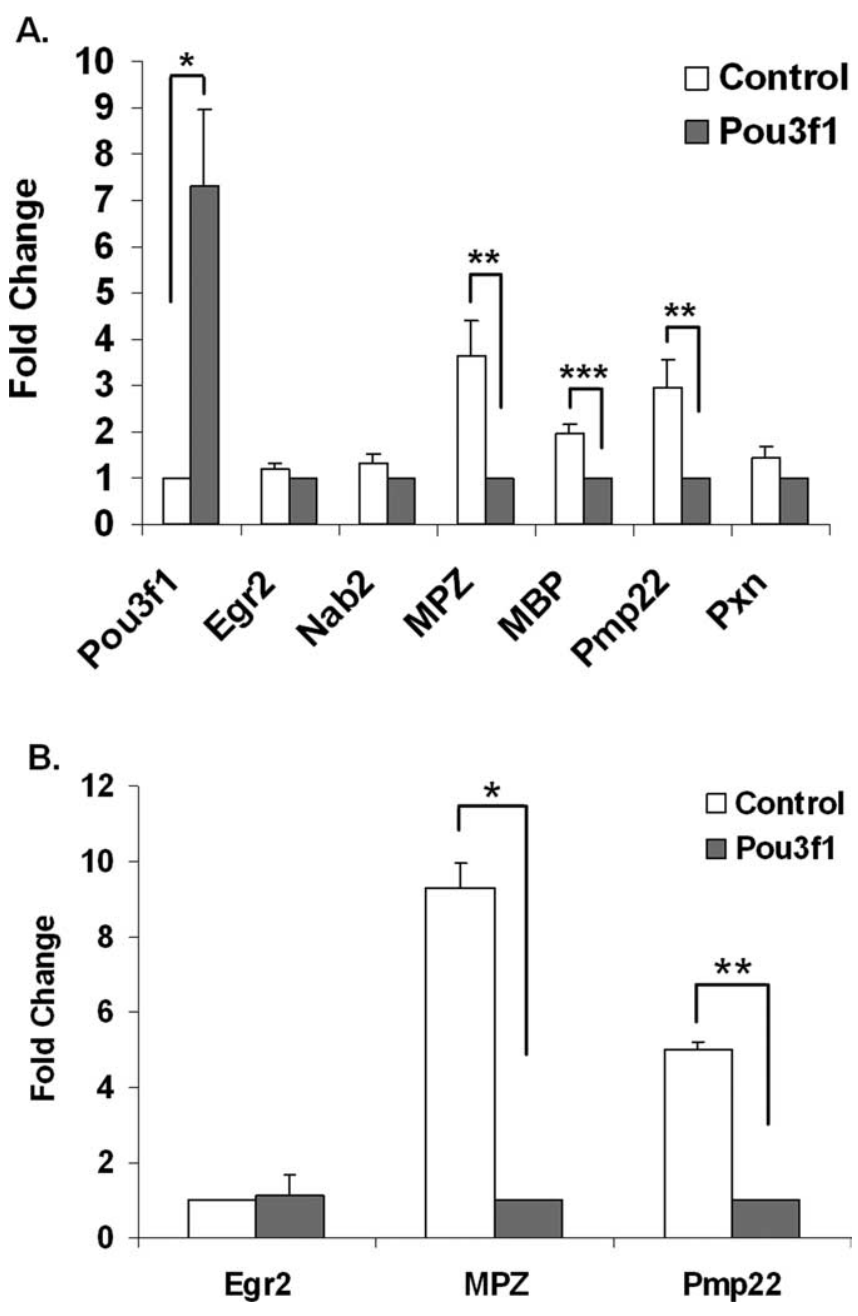

Figure 5. Pou3f1 causes aberrant myelin gene expression despite normal Egr2 levels. $\boldsymbol{A}$, qRT-PCR analysis of gene expression in sciatic nerves from condPou3f1:MPZ(Cre) mice compared with control mice. The condPou3f1:MPZ(Cre) nerves have lower levels of MPZ, MBP, and Pmp22 mRNAs whereas Egr2, Nab2, and periaxin (Pxn) mRNA levels were equivalent. Values are mean $\pm \operatorname{SEM}\left(n=3\right.$ mice of each genotype; ${ }^{*} p<0.0001$; $\left.^{* *} p=0.002 ;{ }^{* * *} p=0.001\right) . \boldsymbol{B}$, Enforced Pou3f1 expression in primary rat Schwann cells via adenovirus infection showed decreased levels of MPZ and Pmp22, whereas Egr2 mRNA levels remained unchanged as measured by $q$ RT-PCR. Values are mean \pm SEM $\left({ }^{*} p=0.0002 ;{ }^{* *} p<0.0001\right)$. Comparable results were achieved in an independent experiment. These in vitro results are consistent with in vivo observations and indicate that Pou3f1 can repress myelin protein gene expression without affecting Egr2 levels. 
deficiency, whereas the normal MPZ and MBP mRNA levels are caused by derepression of these promoters in the absence of Pou3f1. These data support a dual role for Pou3f1 as both an activator and repressor of myelination in that Pou3f1 and other POU proteins may facilitate the transition of a promyelinating to a myelinating Schwann cell by inducing Egr2, while simultaneously withholding the cells from myelination until they are fully ready by repressing MPZ and MBP. The temporal downregulation of Pou3f1 during development would be critical to myelination, because it would allow for the derepression of MPZ and MBP expression. According to this scenario, the continued hypomyelination we observe in condPou3f1:MPZ(Cre) mice would result from the continued repression of myelin protein gene expression. Although defining the complex genetic network required to control the myelination process is still in the early stages, these results point to the need to further elucidate the pathways that lead to Pou3fl downregulation, because it appears to be an important step in promoting proper myelin formation.

\section{References}

Bermingham Jr JR, Scherer SS, O'Connell S, Arroyo E, Kalla KA, Powell FL, Rosenfeld MG (1996) Tst-1/Oct-6/SCIP regulates a unique step in peripheral myelination and is required for normal respiration. Genes Dev 10:1751-1762.

Blanchard AD, Sinanan A, Parmantier E, Zwart R, Broos L, Meijer D, Meier C, Jessen KR, Mirsky R (1996) Oct-6 (SCIP/Tst-1) is expressed in Schwann cell precursors, embryonic Schwann cells, and postnatal myelinating Schwann cells: comparison with Oct-1, Krox-20, and Pax-3. J Neurosci Res 46:630-640.

Brunden KR, Poduslo JF (1987) Lysosomal delivery of the major myelin glycoprotein in the absence of myelin assembly: posttranslational regulation of the level of expression by Schwann cells. J Cell Biol 104:661-669.

Feltri ML, D'Antonio M, Quattrini A, Numerato R, Arona M, Previtali S, Chiu SY, Messing A, Wrabetz L (1999) A novel P0 glycoprotein transgene activates expression of lacZ in myelin-forming Schwann cells. Eur J Neurosci 11:1577-1586.

Feltri ML, Graus Porta D, Previtali SC, Nodari A, Migliavacca B, Cassetti A, Littlewood-Evans A, Reichardt LF, Messing A, Quattrini A, Mueller U, Wrabetz L (2002) Conditional disruption of beta 1 integrin in Schwann cells impedes interactions with axons. J Cell Biol 156:199-209.

Friedrich RP, Schlierf B, Tamm ER, Bosl MR, Wegner M (2005) The class III POU domain protein Brn-1 can fully replace the related Oct-6 during Schwann cell development and myelination. Mol Cell Biol 25:1821-1829.

Ghazvini M, Mandemakers W, Jaegle M, Piirsoo M, Driegen S, Koutsourakis M, Smit X, Grosveld F, Meijer D (2002) A cell type-specific allele of the POU gene Oct-6 reveals Schwann cell autonomous function in nerve development and regeneration. EMBO J 21:4612-4620.

Ghislain J, Charnay P (2006) Control of myelination in Schwann cells: a Krox20 cis-regulatory element integrates Oct6, Brn2 and Sox10 activities. EMBO Rep 7:52-58.

Ghislain J, Desmarquet-Trin-Dinh C, Jaegle M, Meijer D, Charnay P, Frain M (2002) Characterisation of cis-acting sequences reveals a biphasic, axondependent regulation of Krox20 during Schwann cell development. Development 129:155-166.

He X, Treacy MN, Simmons DM, Ingraham HA, Swanson LW, Rosenfeld MG (1989) Expression of a large family of POU-domain regulatory genes in mammalian brain development. Nature 340:35-41.

He X, Gerrero R, Simmons DM, Park RE, Lin CJ, Swanson LW, Rosenfeld MG (1991) Tst-1, a member of the POU domain gene family, binds the promoter of the gene encoding the cell surface adhesion molecule P0. Mol Cell Biol 11:1739-1744.

Herr W, Cleary MA (1995) The POU domain: versatility in transcriptional regulation by a flexible two-in-one DNA-binding domain. Genes Dev 9:1679-1693

Houlden H, Reilly MM (2006) Molecular genetics of autosomal-dominant demyelinating Charcot-Marie-Tooth disease. Neuromolecular Med 8:43-62.

Inoue K, Tanabe Y, Lupski JR (1999) Myelin deficiencies in both the central and the peripheral nervous systems associated with a SOX10 mutation. Ann Neurol 46:313-318.
Jaegle M, Meijer D (1998) Role of Oct-6 in Schwann cell differentiation. Microsc Res Tech 41:372-378.

Jaegle M, Mandemakers W, Broos L, Zwart R, Karis A, Visser P, Grosveld F, Meijer D (1996) The POU factor Oct-6 and Schwann cell differentiation. Science 273:507-510.

Jaegle M, Ghazvini M, Mandemakers W, Piirsoo M, Driegen S, Levavasseur F, Raghoenath S, Grosveld F, Meijer D (2003) The POU proteins Brn-2 and Oct-6 share important functions in Schwann cell development. Genes Dev 17:1380-1391.

Jensen NA, Pedersen KM, Celis JE, West MJ (1998) Neurological disturbances, premature lethality, and central myelination deficiency in transgenic mice overexpressing the homeo domain transcription factor Oct-6. J Clin Invest 101:1292-1299.

Jessen KR, Mirsky R (2002) Signals that determine Schwann cell identity. J Anat 200:367-376.

Le N, Nagarajan R, Wang JY, Araki T, Schmidt RE, Milbrandt J (2005a) Analysis of congenital hypomyelinating Egr2Lo/Lo nerves identifies Sox2 as an inhibitor of Schwann cell differentiation and myelination. Proc Natl Acad Sci USA 102:2596-2601.

Le N, Nagarajan R, Wang JY, Svaren J, LaPash C, Araki T, Schmidt RE, Milbrandt J (2005b) Nab proteins are essential for peripheral nervous system myelination. Nat Neurosci 8:932-940.

Meijer D, Graus A, Kraay R, Langeveld A, Mulder MP, Grosveld G (1990) The octamer binding factor Oct6: cDNA cloning and expression in early embryonic cells. Nucleic Acids Res 18:7357-7365.

Monuki ES, Kuhn R, Weinmaster G, Trapp BD, Lemke G (1990) Expression and activity of the POU transcription factor SCIP. Science 249:1300-1303.

Monuki ES, Kuhn R, Lemke G (1993) Repression of the myelin P0 gene by the POU transcription factor SCIP. Mech Dev 42:15-32.

Nagarajan R, Svaren J, Le N, Araki T, Watson M, Milbrandt J (2001) EGR2 mutations in inherited neuropathies dominant-negatively inhibit myelin gene expression. Neuron 30:355-368.

Peirano RI, Goerich DE, Riethmacher D, Wegner M (2000) Protein zero gene expression is regulated by the glial transcription factor Sox10. Mol Cell Biol 20:3198-3209.

Scherer SS, Xu YT, Nelles E, Fischbeck K, Willecke K, Bone LJ (1998) Connexin32-null mice develop demyelinating peripheral neuropathy. Glia 24:8-20.

Suter U, Moskow JJ, Welcher AA, Snipes GJ, Kosaras B, Sidman RL, Buchberg AM, Shooter EM (1992) A leucine-to-proline mutation in the putative first transmembrane domain of the $22-\mathrm{kDa}$ peripheral myelin protein in the trembler-J mouse. Proc Natl Acad Sci USA 89:4382-4386.

Topilko P, Schneider-Maunoury S, Levi G, Baron-Van Evercooren A, Chennoufi AB, Seitanidou T, Babinet C, Charnay P (1994) Krox-20 controls myelination in the peripheral nervous system. Nature 371:796-799.

Touraine RL, Attie-Bitach T, Manceau E, Korsch E, Sarda P, Pingault V, Encha-Razavi F, Pelet A, Auge J, Nivelon-Chevallier A, Holschneider AM, Munnes M, Doerfler W, Goossens M, Munnich A, Vekemans M, Lyonnet S (2000) Neurological phenotype in Waardenburg syndrome type 4 correlates with novel SOX10 truncating mutations and expression in developing brain. Am J Hum Genet 66:1496-1503.

Warner LE, Mancias P, Butler IJ, McDonald CM, Keppen L, Koob KG, Lupski JR (1998) Mutations in the early growth response 2 (EGR2) gene are associated with hereditary myelinopathies. Nat Genet 18:382-384.

Warner LE, Garcia CA, Lupski JR (1999a) Hereditary peripheral neuropathies: clinical forms, genetics, and molecular mechanisms. Annu Rev Med 50:263-275.

Warner LE, Svaren J, Milbrandt J, Lupski JR (1999b) Functional consequences of mutations in the early growth response 2 gene (EGR2) correlate with severity of human myelinopathies. Hum Mol Genet 8:1245-1251.

Weinstein DE, Burrola PG, Lemke G (1995) Premature Schwann cell differentiation and hypermyelination in mice expressing a targeted antagonist of the POU transcription factor SCIP. Mol Cell Neurosci 6:212-229.

Zorick TS, Syroid DE, Arroyo E, Scherer SS, Lemke G (1996) The transcription factors SCIP and Krox-20 mark distinct stages and cell fates in Schwann cell differentiation. Mol Cell Neurosci 8:129-145.

Zorick TS, Syroid DE, Brown A, Gridley T, Lemke G (1999) Krox-20 controls SCIP expression, cell cycle exit and susceptibility to apoptosis in developing myelinating Schwann cells. Development 126:1397-1406. 